\title{
Mass Loss and Stellar Rotation
}

\author{
Henny J.G.L.M. Lamers \\ Astronomical Institute and SRON Laboratory for Space Research, \\ Utrecht University, Princetonplein 5, NL-3584CC, Utrecht, the \\ Netherlands, lamers@astro.uu.nl
}

\begin{abstract}
The observational evidence for non-spherical winds of rapidly rotating hot and cool stars is presented. Models to explain equatorially enhanced winds of AGB stars and of hot B[e]-supergiants are discussed. We distinguish between models with a disk due to an increased equatorial mass flux and those with a disk due to spherical mass loss with a wind flowing towards the equatorial plane.
\end{abstract}

\section{Introduction}

Fast rotation can increase the mass loss and the angular momentum loss of a star and affect its evolution. In this review I will discuss what is known about the influence of rotation on mass loss, both from observations and theory. (For a comprehensive review of observations and theories of stellar mass loss the reader is referred to the book "Introduction to Stellar Winds" by Lamers \& Cassinelli 1999; herafter called ISW). The role of rotation on the loss of mass and angular momentum of pre-main sequence stars is discussed elsewhere in these proceedings (e.g. by Mathieu and by Wolff et al.) and the Be-stars are discussed by Owocki.

For the study of the evolution with mass loss and with angular momentum loss it is not only important to know that there is (or is not) a density enhancement in the wind near the equator, but it is equally important to know at what stellar lattitude that matter was ejected. Was it ejected at the equator with high specific angular momentum, or was it ejected closer to the pole with lower specific angular momentum and drifted to the equatorial plane?

\section{The Loss of Mass and Angular Momentum from Rotating Stars}

Fast rotation, i.e. with $v_{\text {rot }} \geq 0.5 v_{\text {crit }}$, may change a spherical star with a spherical wind into an equatorially flattened system, with both the radius $R(\theta)$ and the mass flux $F_{m}(\theta)$ (in $\left.\mathrm{g} \mathrm{cm}^{-2} \mathrm{~s}^{-1}\right)$ depending on stellar lattitude $\theta(\theta=0$ at the equator) or co-lattitude $\Theta(\Theta=\pi / 2$ at the equator $)$. The maximum flattening at critical rotation velocity is $R_{\text {eq }} / R_{\text {pole }}=3 / 2$ (e.g. ISW Ch 11). The overall mass loss rate (i.e. from the whole star) is 


$$
\dot{M}=4 \pi \int_{0}^{\pi / 2} F_{m}(\theta) R(\theta)^{2} \cos (\theta) d \theta
$$

(positive by convention) and the angular momentum loss is

$$
d J / d t=-4 \pi \Omega \int_{0}^{\pi / 2} F_{m}(\theta) R^{4}(\theta) \cos ^{3}(\theta) d \theta
$$

For a spherical mass flux, with $R(\theta)=R_{*}$ and $F_{m}(\theta)=F_{m}$, these two equations give the well known results: $\dot{M}=4 \pi R_{*}^{2} F_{m}$ and $d J / d t=-(2 / 3) \dot{M} \Omega R_{*}^{2}$. If the mass is lost only from the equator, i.e. $F_{m}(\theta)$ is a $\delta$-function, the angular momentum loss is

$$
d J / d t=-\dot{M} \Omega R_{\mathrm{eq}}^{2}
$$

The specific angular momentum loss $(d J / d t) / \dot{M}$ is at most 3.4 times as large as for spherical mass loss because $R_{\text {eq }}<1.5 R_{*}$. A higher specific angular momentum loss can only be achieved by magnetic coupling of the wind to the star. This is the case for pre-main sequence stars. A model for magnetically torqued disks of Be stars has recently been proposed by Cassinelli et al. (2002).

In the other extreme: if the mass loss is restricted to a small region near the poles, with $F_{m}(\Theta)=F_{p}$ for $\Theta<\Theta_{p}$ or $\Theta>\pi-\Theta_{p}$ and $F_{m}=0$ outside that range, than $\dot{M}=4 \pi F_{p} R_{p}^{2}\left\{1-\cos \left(\Theta_{p}\right)\right\}$ and $d J / d t=-4 \pi \Omega F_{p} R_{p}^{4}\{(2 / 3)-$ $\left.\cos \left(\Theta_{p}\right)+(1 / 3) \cos ^{3}\left(\Theta_{p}\right)\right\}$, where $\Theta=0$ at the poles. In the limit of very small $\Theta_{p}$ we find that the specific angular momentum loss is

$$
(d J / d t) / \dot{M}=-0.5 \Omega R_{p}^{2} \Theta_{p}^{2}
$$

The specific angular momentum of the star is $J / M_{*}=\Omega R_{\mathrm{gyr}}^{2}$, where $R_{\mathrm{gyr}} \simeq$ $0.5 R_{*}$ is the gyration radius. We see that the mass lost from the poles has a specific angular momentum that is smaller than that of the star so that the star will be spun-up due to polar mass loss.

For fast solidly rotating stars the radiative flux decreases from the pole to the equator proportional to the local gravity (the Von Zeipel effect), so

$$
T_{\mathrm{eff}}^{4}(\theta)=T_{\mathrm{eff}}^{4}(\text { pole })\left\{1-\omega^{2} \cos (\theta)\right\}
$$

where $\omega=v_{\text {rot }}^{\text {eq }} / v_{\text {crit }}$ with $v_{\text {crit }}=\left(G M_{\text {eff }} / R_{\text {eq }}\right)^{1 / 2}$ and $M_{\text {eff }}=M_{*}\left(1-\Gamma_{e}\right)$ where $\Gamma_{e}$ is the Eddington factor (see ISW Ch 11 and Maeder 1999). The pole of a rotating star has a higher effective temperature than the equator. This complicates the calculation of radiation driven winds from rotating stars because the stellar disk is not homogeneous. It also complicates the empirical study of mass loss from rotating stars, because the spectral type of a rotating star will depend on the inclination angle! So if we want to compare mass loss of fast and slow rotating stars, we should compare stars with the same luminosity, mass and volume, and not simply stars with the same spectral type and luminosity class. 


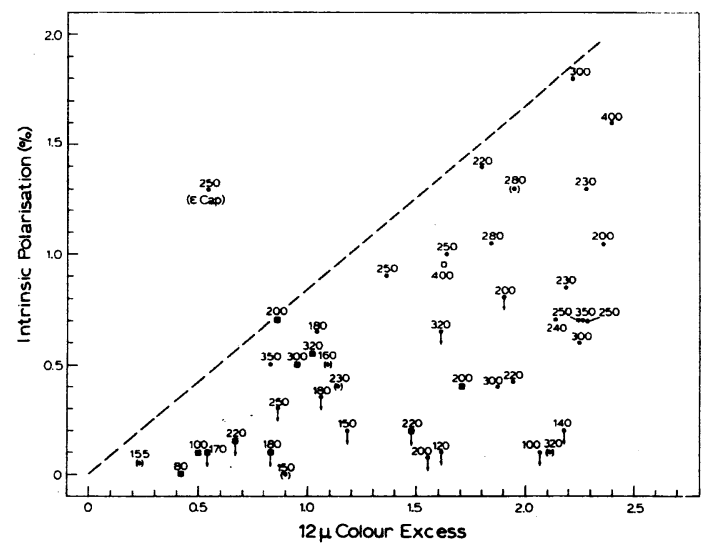

Figure 1. The polarization of Be-stars as a function of the IR-excess shows a triangular diagram because the degree of polarization depends on the optical depth for electron scattering in the circumstellar disks (which scales with the IR-excess) and the viewing angle of the disk. Parameters: $v \sin i$ in $\mathrm{km} \mathrm{s}^{-1}$. (From Coté \& Waters 1987)

\section{Methods for the Determination of Non-Spherical Winds}

\subsection{Direct Imaging}

Direct imaging of stellar winds is only possible for nearby and very extended stars. The winds of some Be-stars and of the hypergiant P Cygni have been measured directly with interferometric techniques by e.g. Quirrenbach et al. (1997), Berio et al. (1999) and Chesneau et al. (2000). They found that the Be-stars $\gamma$ Cas and $\zeta$ Tau show evidence for an equatorial disk with a prograde one-armed oscillation. Images of the winds of cool stars by e.g. Mauron \& Huggins (1999), Tuthill et al. (2000), Weigelt et al. (2002) and others show that the winds of AGB stars are spherically symmetric and that the transition to asymmetric structure occurs very rapidly after the AGB phase (Kwok 1999; Balick \& Frank 2002).

\subsection{Polarimetry}

Polarimetry of hot stars gives direct evidence for the existence of non-spherical circumstellar disks. The polarized radiation is due to the scattering of stellar photons by free electrons in the non-spherical wind. For a rotationally symmetric disk the degree of polarization is maximal if the circumstellar disk is seen edge-on (Brown \& McLean, 1977). The degree of polarization depends on the scattering optical depth $\tau_{e}$ and reaches a maximum of a few $\%$ if $\tau_{e}$ is large in the direction of the plane of the disk (e.g. Magalhães 1992, Schulte-Ladbeck 1994).

Fig. 1 shows the degree of polarization of Be stars as a function of the infrared free-free excess which is a measure of the optical depth of the disk. We see a triangular diagram: the maximum degree of polarization increases with the amount of IR-excess. But for every value of the IR-excess the polarization can reach a range of values, depending on the inclination angle. There is a tendency 

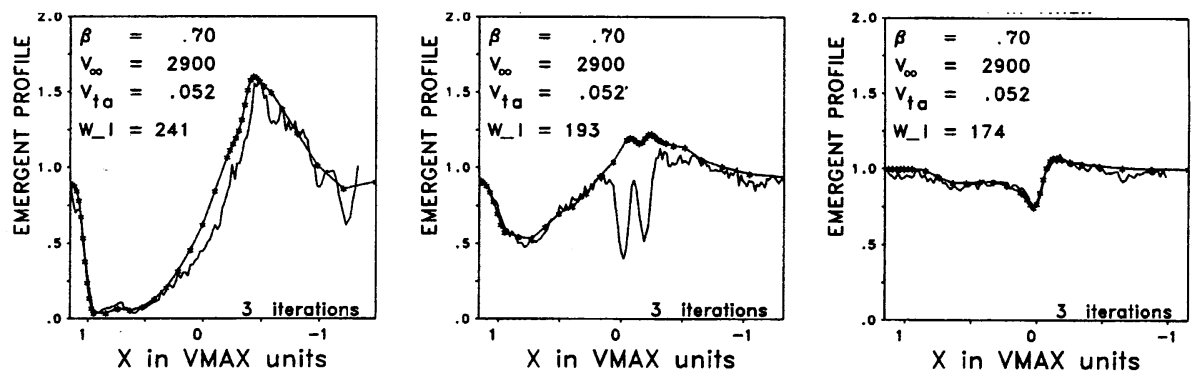

Figure 2. The P Cygni profiles of the NV, CIV and NIV UV lines of the $\mathrm{O} 3$ III $\left(\mathrm{f}^{*}\right)$ star NGC $346, \mathrm{nr} 3$, together with the best fitting theoretical profile. From fits like this the mass flux $F_{m}(\theta=\pi / 2-i)$ in the direction to the observer can be determined. (From Haser et al. 1998)

for the stars with highest $v \sin i$ (i.e. nearly edge-on) to have high polarization, as expected.

\subsection{Methods that Depend on the Column Densities}

Mass loss studies based on blue shifted absorption lines or on the absorption components of P Cygni profiles formed in the wind, yield the column density $N_{i}$ of the wind towards the observer and the profile gives the velocity law $v(r)$ in that direction. The combination of the column density $N_{i}$ and $v(r)$, combined with abundance and ionization structure, gives the mass flux in the direction of the observer. In this way $F_{m}(\theta=\pi / 2-i)$ can be derived. Fig. 2 shows the observed UV P Cygni profiles of an O3 III star and the linefits for a spherical wind model. (The emission component provides little information about the wind structure.)

In principle it should be possible, to determine the $\theta$-dependence of the mass flux from a statistical study of a large sample of nearly identical stars with the same luminosity, mass and mean radius, but different values of $v \sin i$.

\subsection{Methods that Depend on Emission with $\epsilon \sim \rho$}

Mass loss studies based on emission (lines or continuum) from a process that has an emissivity $\epsilon \sim \rho$ are useful for the determination of the total amount of mass around the star. However they do not give much information about the geometrical distribution of this matter. Examples of such processes are: the infrared emission of dust around hot and cool stars and the molecular emission lines in the spectra of cool stars.

The molecular $\mathrm{CO}, \mathrm{OH}$ and $\mathrm{SiO}$ emission lines can be used to derive the amount of mass in the winds of very cool stars. Fig. 3 shows the $\mathrm{CO}$ emission lines of a few AGB stars. The CO $J=1 \rightarrow 0$ line at $2.6 \mathrm{~mm}$ is pumped by IR photons due to dust. Photons at $\lambda=4.6 \mu \mathrm{m}$ excite the $\mathrm{CO}$ molecules from the vibrational ground state $v=0$ to $v=1$ and the subsequent photo-deexcitation gives rise to the emission in rotational transitions in the $v=0$ level (ISW Ch. 2.5). The amount of emission depends linearly on the amount of molecular CO gas around the star (e.g. Knapp \& Morris, 1985). 

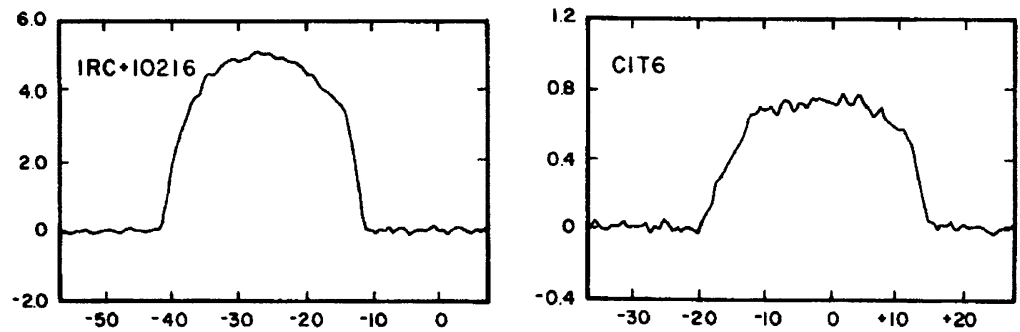

Figure 3. The CO $J=1 \rightarrow 0$ emission lines of two AGB stars. The lines are pumped by IR-emission from dust. These $\mathrm{CO}$ lines can be used to derive the total amount of mass in the wind, but not its spatial distribution. (From Knapp \& Morris, 1985)

\subsection{Methods that Depend on Emission with $\epsilon \sim \rho^{2}$}

The emissivity of processes based on collisional excitation depends on $\rho^{2}$. Examples are the recombination emission lines and the infrared or radio free-free emission of hot star winds. Since $\epsilon \sim \rho^{2}$ the observed emission strength can be used to derive the emission measure of the circumstellar gas $E M=\int \rho^{2} d \mathrm{Vol}$. For winds of fast rotating stars the $E M$ cannot easily be used to derive $F_{m}(\theta)$ or $\dot{M}$ because it requires knowledge of the geometry. A strong emission could be the result of a high mass loss rate or of a small mass loss rate that is highly compressed in the equatorial plane or strongly clumped.

In the studies of the IR free-free excess one usually adopts some velocity function $v(r, \theta)$ and then derives the mass loss rate from the amount of emission. This method has been used for the study of Be stars and B[e] supergiants by e.g. Waters et al. (1987) and Bjorkman (1998) respectively.

The Balmer and other hydrogen emission lines of Be-stars and B[e]-supergiants have been studied by many groups (see e.g. reviews by Stefl (1999) and Zickgraf (1999) and references therein). These lines often show a double peaked emission which is approximately symmetric around the rest wavelength (Fig. 4), indicating the presence of an ionized rotating disk around the star. If the disk is also expanding, the central dip of the profile will be blue-shifted with respect to the emission peaks. The emission profiles from rotating equatorial disks depend strongly on the inclination angle of the rotation axis. If a star is seen pole-on the Balmer lines will not show the characteristic double peaked emission, but a narrow symmetric emission component.

\subsection{Evidence for Disks}

We have seen that different methods for studying mass loss provide different types of information about the stellar wind. The comparison of the results from the different methods gives hints about the geometrical structure. For instance, if the emission measure of the wind, derived from the emission lines or the free-free IR-excess, is considerable higher than predicted from the column density measurement, derived from the $\mathrm{P}$ Cygni profile, with the assumption of spherical mass loss, then one must conclude that the density is enhanced in some regions of the wind. Fig. 5 shows that this is the case for $\mathrm{B}[\mathrm{e}]$-supergiants. If 

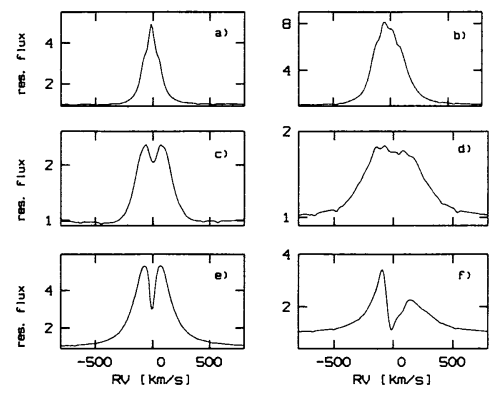

Figure 4. $\mathrm{H} \alpha$ emission profiles of Be-stars show a variety of profiles: from double peaked emissions to asymmetric centrally peaked emission. Double peaked profiles indicate the presence of rotating disks. Single peaked profiles indicate either more-or-less spherical winds or disks seen pole-on. (From Stefl 1999)

the emission lines also show double peaked components due to rotation, one can safely conclude that the high density region is a rotating disk equatorial disk. Whether this disk is expanding or stationary can be derived from the presence or absence of a blue-shifted central absorption of the emission lines.

Many luminous hot stars, e.g B[e]-supergiants and LBVs, have circumstellar dust or molecular gas (see reviews by Eenens and Nota). This also indicates the presence of equatorial disks around these stars, because a disk offers a natural way to shield the molecules or dust from the intense stellar radiation.

We have seen in this section that there is plenty evidence that the winds of rapidly rotating stars are condensed in the equatorial plane. It is still (almost) impossible to decide whether the disk is due to an enhanced equatorial mass flux or due to the wind flowing to the equatorial plane, i.e. wind compression. Therefore the predicted mass flux from rapidly rotating stars is used in evolutionary computations (Maeder \& Meynet 2000; Langer 1998).

\section{Equatorially Enhanced Mass Loss of Dust Driven AGB-Winds}

Images of Planetary Nebulae show clear evidence for a bipolar structure. These nebulae are due to the interaction of the fast wind of their central star (CSPN) and the slow wind of the preceeding AGB phase (Kwok et al. 1978, ISW Ch. 12.5). The asymmetry of the nebulae can be explained by the existence of an equatorially enhanced AGB wind density (Icke 1988, Balick \& Frank 2002). We discuss how the wind density in the equatorial plane of AGB stars can be increased.

The winds of AGB stars are driven by radiation pressure on dust. Since the dust forms only at a distance $R_{\text {dust }} \simeq 1.3$ to $1.5 R_{*}$, the driving of the wind 


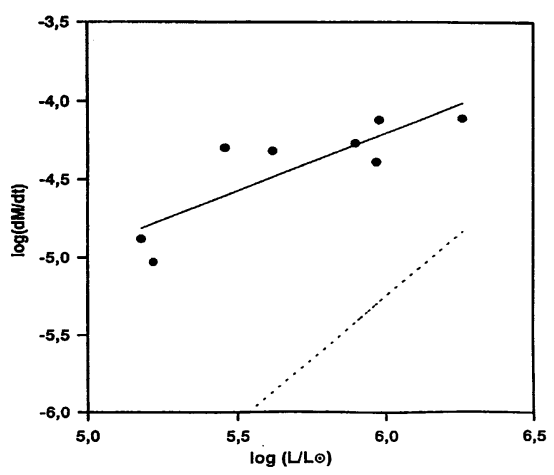

Figure 5. The mass loss rates of $\mathrm{B}[\mathrm{e}]$ supergiants derived from the emission measure of Hydrogen emission lines, under the assumption of spherical symmetry (dots and full line), are much higher than the mass loss rates from stars with similar temperature and luminosity, derived from the column densities of $\mathrm{P}$ Cygni profiles (dashed line). This indicates that the winds of B[e] stars are not spherically symmetric. (From de Freitas Pacheco 1998)

starts only at that distance. The wind quickly reaches its soundspeed close to the dust formation zone, so the mass loss rate is approximately

$$
\dot{M}=4 \pi R_{\text {dust }}^{2} \rho\left(R_{\text {dust }}\right) v_{\text {sound }}\left(R_{\text {dust }}\right)
$$

For a typical AGB-star with $T_{\text {eff }} \simeq 2500 \mathrm{~K}, R_{*} \simeq 300 R_{\odot}$ and $M_{*} \simeq 1 M_{\odot}$ the density scaleheight is about $0.02 R_{*}$, so the dust forms many scaleheights above the photosphere. This implies that the density $\rho\left(R_{\text {dust }}\right)$ will be very small, and hence the mass loss rate will be very small, unless there is a mechanism to increase the density scaleheight significantly.

(1) Dorfi \& Hoefner (1996) have shown that stars at the tip of the AGB have such a small effective gravity that even very slow rotation (with a rotation period of about 10 years!) results in an increase in the equatorial density scaleheight and the equatorial mass flux.

(2) Bowen (1988) and Bowen \& Willson (1991) have shown that the pulsation of AGB and Mira stars results in a "levitation" of the atmosphere because in the outer layers of the atmosphere the fall-back time is longer than the pulsation period. This results in a drastic increase in the density scaleheight so that the density at the dust forming radius and the resulting mass loss rate are increased significantly (Bowen \& Willson 1991; ISW Ch 7). This is shown in Fig. 6. Since the density increase depends strongly on the pulsation amplitude even a small increase in the pulsation amplitude near the equatorial plane of a rotating AGB star will result in an increase in the equatorial mass flux. This possibility has not yet been investigated.

(3) A completely different mechanism for the creation of a dense equatorial wind is the wind compression mechanism in rotating stars (Bjorkman \& Cassinelli 1993). The mass flux is spherically symmetric but the wind gas drifts to the equatorial plane. Ignace et al. (1996) have shown that this also works for rotating AGB stars, see Fig. 7. 


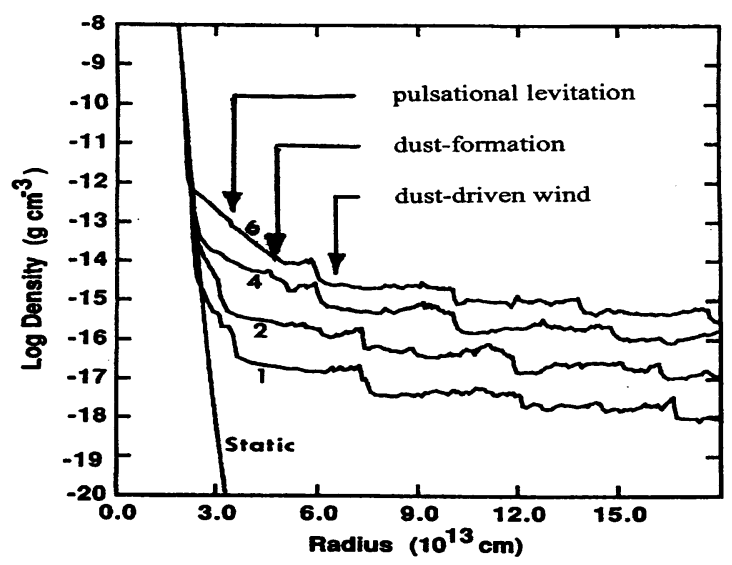

Figure 6. The density structure of the atmosphere of a pulsating AGB star. The steep straight line is the density in case of no pulsation. The four lines show the density as it is modified by the pulsation for different pulsational velocity amplitudes (in $\mathrm{km} \mathrm{s}^{-1}$ ). Dust forms at a distance of about $410^{13}$ $\mathrm{cm}\left(\simeq 2 R_{*}\right)$. The density decrease above that point is due to the wind. The density structure is irregular because it is a snapshot of a time-dependent model. The mass loss rate is proportional to the density at the dust formation radius and is very sensitive to the pulsation amplitude. (adapted from Bowen 1988)

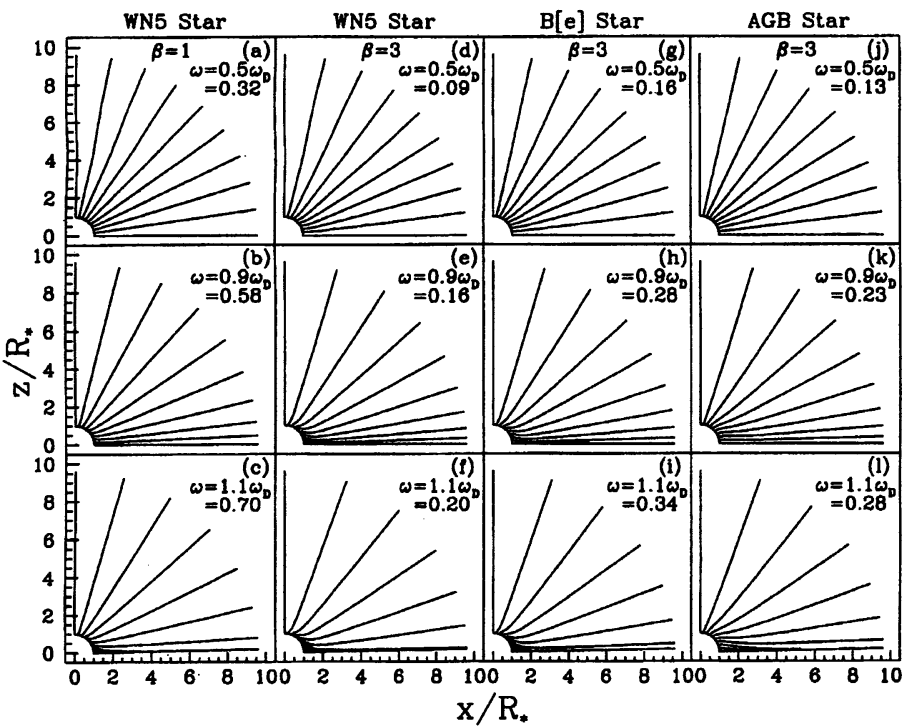

Figure 7. Streamlines in cylindrical coordinates of mass loss from rotating $\mathrm{WN}, \mathrm{B}[\mathrm{e}]$ and AGB-stars. For rapidly rotating stars the gas moves towards the equatorial plane where it produces a density enhancement: i.e. a wind compressed disk or zone. The dimensionless parameter is $\omega=v_{\text {rot }} / v_{\text {crit }}$. (From Ignace et al. 1996) 

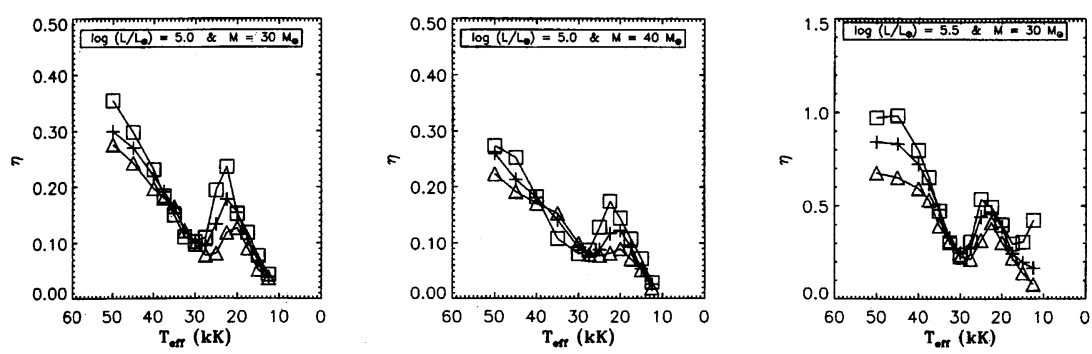

Figure 8. The predicted dependence of the wind momentum efficiency $\dot{M} v_{\infty} /(L / c)$ as a function of $T_{\text {eff }}$ for stars with $M=30$ and $40 M_{\odot}$, $\log \left(L / L_{\odot}\right)=5.0$ and 5.5 and with $Z=0.02$. The adopted terminal velocity is $v_{\infty} / v_{\text {esc }}=1.3$ (squares), 2.0 (plusses) or 2.6 (triangles). Notice the bi-stability jump around $T_{\text {eff }} \simeq 25000 \mathrm{~K}$. (From Vink et al. 2000)

\section{Equatorially Enhanced Mass Loss of Line Driven Winds from Hot Stars}

In order to understand the effect of rotation on the winds of hot stars we first have to consider the winds of non-rotating hot stars. The winds of hot stars are driven by radiation pressure in spectral lines (Castor et al. 1975; Kudritzki \& Puls 2000). Vink et al. (2000 and 2001) have computed mass loss rates for a large grid of stellar models using non-LTE populations and multiple scattering of photons. This results in expressions for the predicted mass loss rates of hot stars for various metallicities which can be used in evolutionary models. Fig. 8 shows the results in terms of the momentum transfer efficiency $\eta=\dot{M} v_{\infty} /(L / c)$, for one particular set of models, defined by $L, M$ and $Z$ as a function of $T_{\text {eff }}$. Notice the "bi-stability jump" near $T_{\text {eff }} \simeq 25000 \mathrm{~K}$, where a change in the ionization in the lower part of the wind occurs. When $T_{\text {eff }}$ drops below about $25000 \mathrm{~K}$, the mass loss increases by about a factor four and the wind velocity decreases by a factor two so that the density of the wind increases by an order of magnitude (Pauldrach \& Puls 1990; Lamers et al. 1995; Vink et al. 1999). Observations show that the jump occurs at a temperature of about $21000 \mathrm{~K}$, rather than $25000 \mathrm{~K}$. This could be due to imperfections in the calculations of the ionization balance in the models (Vink et al. 1999). The temperature of the jump will not be the same for all stars as it depends on the ionization and hence on the density and the local gravity (Vink et al. 2000). We assume here that the jump occurs somewhere in the range of $21000<T_{\text {jump }}<25000 \mathrm{~K}$.

Let us now consider the effect of fast rotation on a line driven wind. The two dominant effects of fast solid rotation are: (a) the reduction of the effective surface gravity from pole to equator due to the centrifugal force and rotational flattening and (b) the decrease of the radiative flux from pole to equator due to the Von Zeipel effect. The radiative flux scales proportional to the local net gravity $F_{\text {rad }}(\theta) \propto g_{\text {net }}=\left\{G M\left(1-\Gamma_{e}\right) / R^{2}(\theta)\right\}\left\{1-\omega^{2} \sin \theta^{2}\right\}$, where $\omega=$ $v_{\text {rot }}^{\mathrm{eq}} / v_{\text {crit }}$. The mass flux of a line driven wind scales approximately as $F_{m} \propto$ $F_{\text {rad }}^{1 / \alpha^{\prime}} \times g_{\text {net }}^{\left(\alpha^{\prime}-1\right) /\left(\alpha^{\prime}\right)}$, with $\alpha^{\prime} \simeq 0.5$ (Kudritzki \& Puls 2000). So $F_{m}(\theta) \propto g_{\text {net }}(\theta)$. The terminal velocity of the wind scales with the local escape velocity. This results in approximately the following dependence on stellar lattitude 

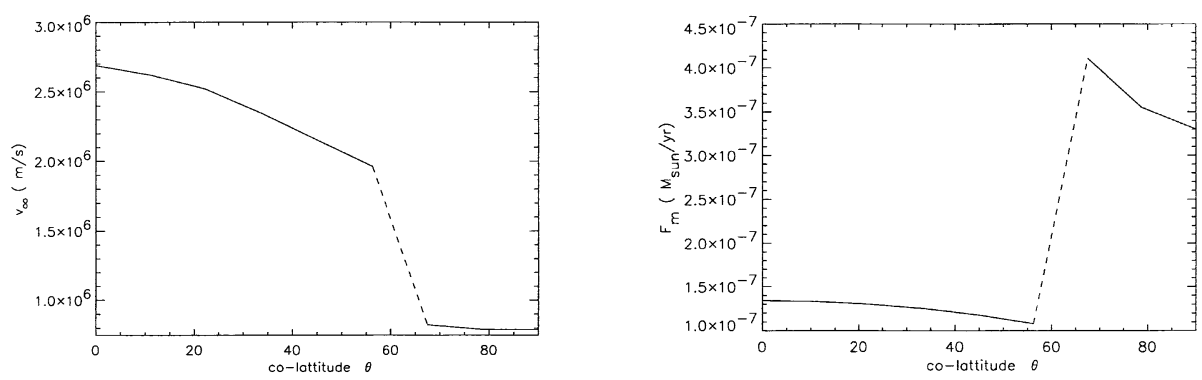

Figure 9. A rotation induced bi-stability model of a $\mathrm{B}[\mathrm{e}]$-supergiant. The lattitude dependence of the terminal wind velocity (left) and mass flux (right), expressed as $4 \pi R_{*}^{2} F_{m}$ in $M_{\odot} \mathrm{yr}^{-1}$, from pole $\Theta=0$ to equator $\Theta=90$ of a star of $L=10^{5} L_{\odot}, M=17.5 M_{\odot}$ and $R_{\text {pole }}=17 R_{\odot}$ rotating at $60 \%$ of the critical velocity. Notice the bi-stability jump at $\Theta \simeq 60$ degrees, where the mass flux increases and the wind velocity decreases drastically. This results in an equatorial disk. (From Pelupessy et al. 2000)

$$
\begin{aligned}
& F_{m}(\theta) \propto g_{\mathrm{net}}(\theta) \propto\left(1-\omega^{2} \cos ^{2} \theta\right) / R^{2}(\theta) \\
& v_{\infty}(\theta) \propto v_{\mathrm{esc}}(\theta) \propto \sqrt{\left(1-\omega^{2} \cos ^{2} \theta / R(\theta)\right.} \\
& \rho(r, \theta) \propto F_{m}(\theta) v(r, \theta)^{-1}(r / R(\theta))^{-2} \propto \sqrt{R(\theta)\left(1-\omega^{2} \cos ^{2} \theta\right)}
\end{aligned}
$$

Based on these simple expressions we expect a higher wind density at the poles $(\cos \theta=0)$ than at the equator. This has been confimed by detailed calculations by Petrenz \& Puls (1996) and Pelupessy et al. (2000) who took into account the non-spherical brightness distribution of the star in the calculation of the radiation pressure. However the observations clearly show that $\mathrm{B}[\mathrm{e}]$ supergiants have equatorial disks (see contributions by Eenens, Nota and Kraus \& Lamers, these proceedings).

The disks of $\mathrm{B}[\mathrm{e}]$ stars can be explained by taking into account the rotation induced bi-stability jump of radiation driven winds, as originally proposed by Lamers \& Pauldrach (1991). The winds of fast rotating B-stars with an effective temperature higher than $T_{\text {jump }}$ at the pole and lower than $T_{\text {jump }}$ at the equator will have a bi-stability jump at some intermediate stellar lattitude $\theta_{\text {jump }}$. At $|\theta|<\theta_{\text {jump }}$ the mass flux will be higher, the wind velocity will be lower and the density will be higher than at higher lattitudes $|\theta|>\theta_{\text {jump. This results in an }}$ equatorial disk. This is confirmed by detailed calculations by Pelupessy et al. (2000) who used the bi-stability parameters calculated by Vink et al. (2000) (see Fig. 9). If disk-compression occurs, as suggested by Bjorkman \& Cassinelli (1993) and Bjorkman (1998), then the disks of B[e]-stars formed by rotation induced bi-stability may be compressed to even higher density (ISW Ch 11). 


\section{Summary and Conclusions}

1. There is both direct and indirect observational evidence that fast rotation affects the mass loss rates of stars during and after the main sequence phase:

(a) direct imaging of a few nearby Be stars,

(b) polarization due to electron scattering in the asymmetric winds of hot stars,

(c) double peaked emission profiles of Balmer and Paschen lines of classical Bestars,

(d) the high emission measure derived from the emission lines and free-free excess of $\mathrm{Be}$ and $\mathrm{B}[\mathrm{e}]$ stars,

(e) the presence of molecular gas and dust in the immediate vicinity of $\mathrm{B}[\mathrm{e}]$ stars and the LBV AG Car requires shielding by a disk,

(f) the bi-polar symmetry of both planetary nebulae and nebulae around massive stars.

2. To understand the evolution of rotating stars it is important to know how much angular momentum is lost due to stellar winds. If the observed equatorial disks are due to spherical mass loss with the gas flowing towards the equatorial region (i.e. the wind compressed disk or wind compressed zone mechanism), then the loss of angular momentum is much less than for a disk formed by an enhanced equatorial mass flux.

3. The empirical determination of the dependence of the mass flux and the wind velocity on stellar lattitude is difficult because absorption lines only give information about a particular direction, viz. towards the observer, whereas emission lines give information about the total amount of mass or the total emission measure irrespective of its geometrical distribution.

4. Dust driven winds of cool stars can be rotationally enhanced by an increase in the equatorial density scaleheight at the layers between the photosphere and the dust-formation radius. This could be due to either rotation alone (at the tip of the AGB) or due to an increase in the equatorial pulsational amplitude. Alternatively, the equatorial density may be enhanced by wind compression.

5. Line driven winds of hot stars can have disks due to rotation induced bistability, e.g. in B[e] supergiants. This mechanism explains in a natural way why such disks only occur around spectral type B where the bi-stability jump occurs. The density in these disks might be further increased by wind compression.

\section{References}

Balick, B. \& Frank, A. 2002, ARAA 40, 439

Berio, P., Stee, Ph., Vakili, F. et al. 1999, A\&A 345, 203

Bjorkman, J.E. 1998, in B[e] stars, A.M. Hubert \& C. Jaschek (eds), Kluwer Acad. Publ., 189

Bjorkman, J.E. \& Cassinelli, J.P. 1993, ApJ 409, 429

Bowen, G.H. 1988, ApJ 329, 299

Bowen, G.H. \& Willson, L.A. 1991, ApJ 375, L53

Brown, J.C. \& McLean, I.S. 1977, A\&A 57, 141

Cassinelli, J.P., Brown, J.C., Maheswaran, M., Miller N.A. \& Telfer, D.C 2002, ApJ 578,951

Castor, J I., Abbott, D.C. \& Klein, R.I. 1975, ApJ 195, 157 
Chesneau, O., Roche, M, Boccaletti, A. et al. 2000, A\&AS 144, 523

Coté, J. \& Waters, L.B.F.M. 1987, A\&A 176, 93

de Freitas Pacheco, J.A. 1998, in B[e] stars, A.M. Hubert \& C. Jaschek (eds), Kluwer Acad. Publ., 221

Dorfi, E.A. \& Hoefner, S. 1966, A\&A 313, 605

Haser, S.M., Pauldrach, A.W.A., Lennon, D.J. et al. 1998, A\&A 330, 285

Icke, V. 1988, A\&A 202, 177

Ignace, R., Cassinelli, J.P. \& Bjorkman, J.E. 1996, ApJ 459, 671

Knapp, G.R. \& Morris, M., 1985, ApJ 292, 640

Kudritzki, R.P, \& Puls, J. 2000, ARAA 38, 613

Kwok, S., Purton, C.R. \& FitzGerald, P.M. 1978, ApJ 219, L125

Kwok, S., Volk, K. \& Hrivnak, B.J. 2002, ApJ 573, 720

Lamers, H.J.G.L.M. \& Cassinelli, J.P. 1999, Introduction to Stellar Winds, Cambridge University Press (ISW).

Lamers, H.J.G.L.M. \& Pauldrach, A.W.A. 1991, A\&A 244, L5

Lamers, H.J.G.L.M., Snow, T.P. \& Lindholm, D.M. 1995, ApJ 455, 269

Langer, N. 1998, A\&A 329, 551

Maeder, A. 1999, in Variable and non-spherical winds in hot luminous stars, B. Wolf, O.Stahl \& A. Fullerton (eds), Springer Verlag, Berlin

Maeder, A. \& Meynet, G. 2000, A\&A 361, 159

Magalhaes, A.M. 1992 ApJ 398, 286

Mauron, N. \& Huggins, P.J. 1999, A\&A 349, 203

Pauldrach, A.W.A. \& Puls, J. 1990, A\&A 237, 409

Pelupessy, I., Lamers, H.J.G.L.M. \& Vink, J.S. 2000, A\&A 359, 695

Petrenz, P. \& Puls, J. 1996, A\&A 312, 195

Quirrenbach, A., Bjorkman, K.S., Bjorkman, J.E. et al. 1997, ApJ 479, 477

Schulte-Ladbeck, R.E. 1994, Ap\&SS 221, 347

Stefl, S. 1999, in Variable and non-spherical winds of luminous hot stars, B. Wolf, O. Stahl \& A.W. Fullerton (eds), Springer Verlag, 40

Tuthill, P.G., Monnier, J.D., Danchi, W.C. \& Lopez, B. 2000, ApJ 543, 284

Vink, J.S., de Koter, A. \& Lamers, H.J.G.L.M. 1999, A\&A 350, 181

Vink, J.S., de Koter, A. \& Lamers, H.J.G.L.M. 2000, A\&A 362, 295

Vink, J.S., de Koter, A. \& Lamers, H.J.G.L.M. 2001, A\&A 369, 574

Waters, L.B.F.M., Coté, J. \& Lamers, H.J.G.L.M. 1987, A\&A 185, 206

Weigelt, G., Balega, I.I., Bloecker, T. et al. 2002, A\&A 392, 131 